\title{
DYNAMICAL SUSCEPTIBILITIES IN THE STRONG COUPLING APPROACH: A GENERAL SCHEME AND THE FALICOV-KIMBALL MODEL
}

\author{
A. M. Shvaika \\ Institute for Condensed Matter Physics, National Academy of Sciences of Ukraine \\ 1 Svientsitskii Str., Lviv, UA-79011, Ukraine
}

(Received August 30, 2001; received in final form September 27, 2001)

\begin{abstract}
A general scheme to calculate dynamical susceptibilities of strongly correlated electron systems within the dynamical mean field theory is developed. The approach is based on an expansion over electron hopping around the atomic limit (within the diagrammatic technique for site operators: projection and Hubbard ones) in infinite dimensions. As an example, the Falicov-Kimball and simplified pseudospin-electron models are considered for which an analytical expressions for dynamical susceptibilities are obtained.

Key words: dynamical susceptibilities, dynamical mean field theory, Falicov-Kimball model, pseudospin-electron model.
\end{abstract}

PACS number(s): 75.40.Gb, 71.10.Fd, 71.27.+a

\section{INTRODUCTION}

The development of the Dynamical Mean Field Theory (DMFT), which is exact in the $d=\infty$ limit, clarified some problems connected with the simultaneous consideration of the electron hopping and strong local correlations and stimulated a large progress in the understanding of the strongly correlated electron systems [1]. It was shown by Metzner and Vollhardt $[2,3]$ that in the $d=\infty$ limit self-energies are single-site quantities (do not depend on wave vector) which leads to a significant simplification. But such a local self-energy only probes local properties in this limit and cannot detect instabilities associated with a specific wave-vector [1] that requires the calculation of susceptibilities.

An analytical scheme developed for the descriptions of the strongly correlated electrons can be divided into two types: (i) weak-coupling theories, that are based on the expansion over local many-electron interactions (Dyson approach) and are close to the standard Fermiliquid theory, and (ii) strong coupling theories, that start from the expansion over electron hopping around the atomic limit (see Ref. [4] and references therein). In the weak-coupling approach susceptibilities are solutions of the Bethe-Salpeter equation and it is known that in the $d \rightarrow \infty$ limit the corresponding irreducible four-vertices are also local $[1,5]$.

The aim of this article is to develop a general scheme to calculate dynamical susceptibilities within a strongcoupling DMFT approach for strongly correlated electron systems. A special case of the binary alloy [FalicovKimball (FK)] type models, that can be solved exactly in the limit of infinite dimensions [6], will be also considered. Static susceptibilities for the FK model have been already investigated by Brandt and Mielsch [6], who found an Ising-like phase transition to a chessboard charge-density-wave phase at half filling, and Freericks [7], who showed that system also displayed the incommensurate order and phase separation at other fillings.
The preliminary short version of this article was published in the Proceedings of M2S-HTSC-VI Conference [8]. Here we present the details of the general scheme.

\section{TWO APPROACHES IN MANY-ELECTRON THEORY}

In general, the Hamiltonian of the electronic system with strong local correlations can be written in the following form

$$
H=H_{t}+H_{\mathrm{loc}}
$$

where

$$
H_{t}=\sum_{i j \sigma} t_{i j} a_{i \sigma}^{\dagger} a_{j \sigma}
$$

describes an intersite electron hopping and $H_{\mathrm{loc}}$ is a sum of the single site Hamiltonians

$$
H_{\mathrm{loc}}=\sum_{i} H_{i}
$$

which describe local electron correlations and/or interaction with other local excitations (lattice vibrations, (pseudo)spins, etc.).

\section{A. Weak-coupling approach}

As a rule, the first term in (2.1) is considered as an initial (zero-order) Hamiltonian and the second one is considered as a perturbation. Such an approach is well known as the Dyson weak-coupling approach, where the single electron Green's functions are determined by the Dyson equation

$$
G_{\sigma k}\left(\omega_{\nu}\right) \equiv \longrightarrow=\longrightarrow+\longrightarrow
$$




\section{A. M. SHVAIKA}

or

$$
\begin{aligned}
G_{\sigma \boldsymbol{k}}\left(\omega_{\nu}\right) & =\mathcal{G}_{\sigma \boldsymbol{k}}\left(\omega_{\nu}\right)+\mathcal{G}_{\sigma \boldsymbol{k}}\left(\omega_{\nu}\right) \Sigma_{\sigma \boldsymbol{k}}\left(\omega_{\nu}\right) G_{\sigma \boldsymbol{k}}\left(\omega_{\nu}\right) \\
& =\frac{1}{i \omega_{\nu}+\mu-t_{\boldsymbol{k}}-\Sigma_{\sigma \boldsymbol{k}}\left(\omega_{\nu}\right)} .
\end{aligned}
$$

Here

$$
\mathcal{G}_{\sigma \boldsymbol{k}}\left(\omega_{\nu}\right)=\frac{1}{i \omega_{\nu}+\mu-t_{\boldsymbol{k}}}
$$

and the self-energy $\Sigma_{\sigma \boldsymbol{k}}\left(\omega_{\nu}\right)$ describes all scattering processes originated from the second term in (2.1) and cannot be divided into parts by cutting off one zero-order Green's function $\mathcal{G}_{\sigma \boldsymbol{k}}\left(\omega_{\nu}\right)$ line.

Also, it is well known that within such a weak-coupling approach the electron susceptibilities (charge, spin, etc.) can be presented in the form

$$
\chi_{\boldsymbol{q}}^{A B}\left(\omega_{m}\right)=-\longleftrightarrow+
$$

where the type of susceptibility is determined by the ending parts. The full four vertex is a solution of the BetheSalpeter equation

$$
D=D-D\left[\begin{array}{l}
D \\
D
\end{array}\right.
$$

with irreducible four vertex $D$ that, in a same way as self-energy, describes scattering processes originated from local correlations and cannot be divided into parts by cutting off two zero-order Green's function lines.

\section{B. Strong-coupling approach}

On the other hand, an alternative approach based on the expansion over electron hopping around the strongcoupling limit can be built $[9,10]$. In this case, the singleelectron Green's functions are determined by the Larkin equation $[9,10]$

$$
G_{\sigma k}\left(\omega_{\nu}\right) \equiv \rightarrow=-\Xi-D^{-\Xi} \sim \sim \longrightarrow
$$

or

$$
\begin{aligned}
G_{\sigma \boldsymbol{k}}\left(\omega_{\nu}\right) & =\Xi_{\sigma \boldsymbol{k}}\left(\omega_{\nu}\right)+\Xi_{\sigma \boldsymbol{k}}\left(\omega_{\nu}\right) t_{\boldsymbol{k}} G_{\sigma \boldsymbol{k}}\left(\omega_{\nu}\right) \\
& =\frac{1}{\Xi_{\sigma \boldsymbol{k}}^{-1}\left(\omega_{\nu}\right)-t_{\boldsymbol{k}}},
\end{aligned}
$$

where $\Xi_{\sigma \boldsymbol{k}}\left(\omega_{\nu}\right)$ is an irreducible according to Larkin part which cannot be divided into parts by cutting off one hopping (wavy) line.

Now, susceptibilities can be presented in the following form

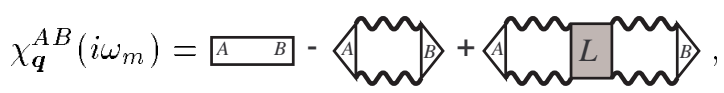

where

$$
m=m+m \rightarrow m
$$

is a sum of the chains of hopping lines and full four vertex is a solution of the equation

$$
L=L-L C L \text {. }
$$

In Eqs. (2.11) and (2.13) quantities $\square,\langle$ and $L$ are irreducible verticies which cannot be divided into parts by cutting off two wavy (hopping) lines. It should be noted that, in contrast to the weak-coupling approach, vertices in the strong-coupling approach correspond to irreducible many-particle Green's functions.

\section{Connection between approaches}

From Eqs. (2.5) and (2.10) one can get a connection between the self-energy and the irreducible vertex function according to the Larkin equation:

$$
\Xi_{\sigma \boldsymbol{k}}^{-1}\left(i \omega_{\nu}\right)=i \omega_{\nu}+\mu-\Sigma_{\sigma \boldsymbol{k}}\left(i \omega_{\nu}\right) .
$$

The connection between the four vertices in the weak and strong coupling approaches is more complicated. For the full four verticies one can get

$$
L={ }_{-}^{-}|D|^{\mathrm{D}-},
$$

whereas the irreducible four verticies are connected by equation

$$
L={ }_{-a}^{-D^{\prime}}{ }^{\mathrm{D}-},
$$

where $D^{\prime}$ is a solution of equation

$$
D^{\prime}=D-D \cdot D^{\prime} .
$$

Here

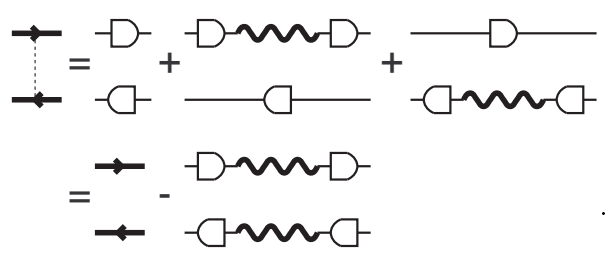

In the case when the operators $\hat{A}$ and $\hat{B}$ are constructed only by the electronic operators, then for three and two verticies we find

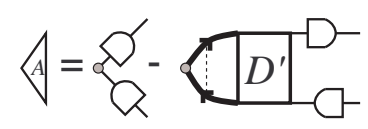

and

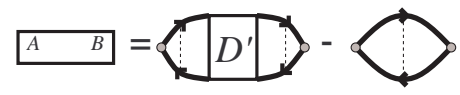

which can be treated as some kind of sum rules. 


\section{DYNAMICAL MEAN FIELD THEORY}

In the case of infinite dimensions $d \rightarrow \infty$ one should scale the hopping integral according to

$$
t_{i j} \rightarrow \frac{t_{i j}}{\sqrt{d}}
$$

in order to obtain a finite density-of-states and it was shown by Metzner [3] that in this limit the irreducible part becomes local

$$
\Xi_{i j \sigma}\left(\tau-\tau^{\prime}\right)=\delta_{i j} \Xi_{\sigma}\left(\tau-\tau^{\prime}\right)
$$

or

$$
\Xi_{\sigma \boldsymbol{k}}\left(\omega_{\nu}\right)=\Xi_{\sigma}\left(\omega_{\nu}\right)
$$

Such a site-diagonal function, as it was shown by Brandt and Mielsch [6], can be calculated by mapping the $d \rightarrow \infty$ lattice problem (2.1) with intersite hopping (3.1) onto the atomic model with single-site hopping

$$
H_{t} \rightarrow \int_{0}^{\beta} d \tau \int_{0}^{\beta} d \tau^{\prime} \sum_{\sigma} \zeta_{\sigma}\left(\tau-\tau^{\prime}\right) a_{\sigma}^{\dagger}(\tau) a_{\sigma}\left(\tau^{\prime}\right)
$$

Here $\zeta_{\sigma}\left(\tau-\tau^{\prime}\right)$ is the auxiliary Kadanoff-Baym field (dynamical mean field) which has to be self-consistently determined from the condition that the same function $\Xi_{\sigma}\left(\omega_{\nu}\right)$ defines the Green's function for the lattice and atomic model. The self-consistent set of equations for $\Xi_{\sigma}\left(\omega_{\nu}\right)$ and $\zeta_{\sigma}\left(\omega_{\nu}\right)$ (e. g., see Ref. [1] and references therein) is the following:

$$
\begin{aligned}
\frac{1}{N} \sum_{\boldsymbol{k}} \frac{1}{\Xi_{\sigma}^{-1}\left(\omega_{\nu}\right)-t_{\boldsymbol{k}}} & =\frac{1}{\Xi_{\sigma}^{-1}\left(\omega_{\nu}\right)-\zeta_{\sigma}\left(\omega_{\nu}\right)} \\
& =G_{\sigma}^{(a)}\left(\omega_{\nu},\left\{\zeta_{\sigma}\left(\omega_{\nu}\right)\right\}\right)
\end{aligned}
$$

where $G_{\sigma}^{(a)}\left(\omega_{\nu},\left\{\zeta_{\sigma}\left(\omega_{\nu}\right)\right\}\right)$ is the Green's function for the atomic model (3.4).

In the same way as it was done by Metzner [3] for the irreducible part $\Xi_{\sigma}\left(\omega_{\nu}\right)$ one can prove that in the $d \rightarrow \infty$ limit all irreducible verticies in the strong-coupling approach (Eqs. (2.11) and (2.13)) are also single site quantities. So, they can be also be calculated from the atomic model. On the other hand, using Eqs. (2.14)-(2.20) it is easy to show that the self-energy and irreducible verticies in the weak-coupling approach are also local quantities (in Refs. [5] that was proved using a different approach).

In order to calculate these single-site irreducible verticies first of all one has to calculate an irreducible many particle Green's functions for atomic model (3.4), i. e.,

$$
\begin{aligned}
{ }_{2}^{1} \mathcal{O}_{3}^{4} & \equiv\left\langle T a_{1}^{\dagger} a_{2} a_{3}^{\dagger} a_{4}\right\rangle_{a} \\
& -\left\langle T a_{1}^{\dagger} a_{2}\right\rangle_{a}\left\langle T a_{3}^{\dagger} a_{4}\right\rangle_{a}+\left\langle T a_{1}^{\dagger} a_{4}\right\rangle_{a}\left\langle T a_{3}^{\dagger} a_{2}\right\rangle_{a}, \\
& \bigsqcup_{2}^{1} \equiv\left\langle T \hat{A}_{0} a_{1}^{\dagger} a_{2}\right\rangle_{a}-\left\langle\hat{A}_{0}\right\rangle_{a}\left\langle T a_{1}^{\dagger} a_{2}\right\rangle_{a}
\end{aligned}
$$

and

$$
{ }_{0} \mathrm{~A} \quad \mathrm{~B}{ }_{1} \equiv\left\langle T \hat{A}_{0} \hat{B}_{1}\right\rangle_{a}-\left\langle\hat{A}_{0}\right\rangle_{a}\left\langle\hat{B}_{1}\right\rangle_{a} .
$$

Then irreducible four vertex $D$ for the weak-coupling approach can be obtained from equation (2.8), where now arrows indicate single-electron Green's functions for the atomic model (3.5) and full four vertex is determined from the many-particle Green's function by

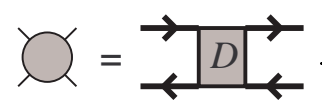

On the other hand, irreducible four vertex $L$ for the strong-coupling approach can be obtained from equation (2.13), where the full four vertex is determined from the many-particle Green's function by

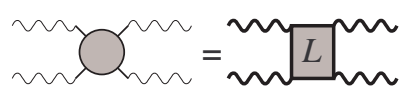

or

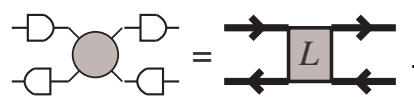

Now, the thin wavy lines are the dynamical mean field $\zeta_{\sigma}\left(\omega_{\nu}\right)$ and the thick ones, in contrast to $(2.12)$, represent the sum of chains of wavy lines

$$
\tilde{\zeta}_{\sigma}\left(\omega_{\nu}\right)=\frac{\zeta_{\sigma}\left(\omega_{\nu}\right)}{1-\Xi_{\sigma}\left(\omega_{\nu}\right) \zeta_{\sigma}\left(\omega_{\nu}\right)}=\zeta_{\sigma}\left(\omega_{\nu}\right) \frac{G_{\sigma}^{(a)}\left(\omega_{\nu}\right)}{\Xi_{\sigma}\left(\omega_{\nu}\right)} .
$$

From the expression for the irreducible many particle Green's function (3.7), (3.8) and (3.6) for atomic model one can find irreducible verticies $\langle$ and $\square$ by equations

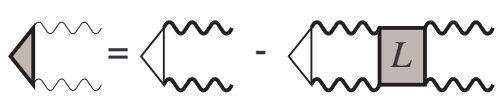

and

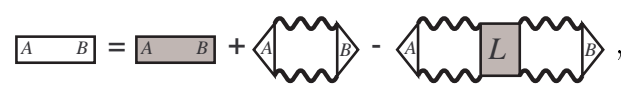

respectively, that complete the calculation of the irreducible verticies for the dynamical susceptibilities.

Finally, for the lattice dynamical susceptibility in the weak (2.7) and strong (2.11) coupling approaches we get

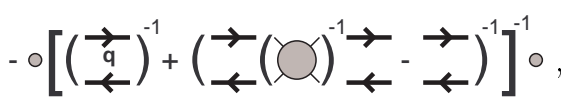

and

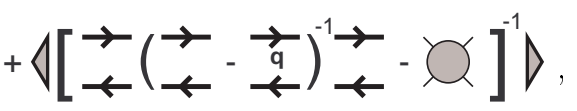

respectively. Here, $(\ldots)^{-1}$ denote an inverse kernels of the corresponding integral equations,

$$
\stackrel{\leftarrow}{\leftarrow}=G_{\sigma}^{(a)}\left(\omega_{\nu}\right) G_{\sigma}^{(a)}\left(\omega_{\nu+m}\right)
$$

and

$$
\stackrel{\leftrightarrow}{\leftarrow}=\frac{1}{N} \sum_{\boldsymbol{k}} G_{\sigma \boldsymbol{k}}\left(\omega_{\nu}\right) G_{\sigma \boldsymbol{k}+\boldsymbol{q}}\left(\omega_{\nu+m}\right) .
$$




\section{A. M. SHVAIKA}

\section{BINARY ALLOY TYPE MODELS}

To test the possibilities of the above approach we consider a binary alloy type model

$$
H_{i}=\frac{g}{2}\left(P_{i}^{+}-P_{i}^{-}\right) n_{i}-\frac{h}{2}\left(P_{i}^{+}-P_{i}^{-}\right),
$$

where $P_{i}^{ \pm}=\frac{1}{2} \pm S_{i}^{z}$ for the $U=0$ pseudospin-electron (PE) model [11], $P_{i}^{+}=c_{i}, P_{i}^{-}=1-c_{i}$ for a binary alloy, and $a_{i \sigma} \rightarrow d_{i}, n_{i}=d_{i}^{\dagger} d_{i}, P_{i}^{+}=f_{i}^{\dagger} f_{i}, P_{i}^{-}=1-f_{i}^{\dagger} f_{i}$ for the Falicov-Kimball (FK) model [12]. The main difference between these models is in the way that the averaging procedure is performed (a statistical one for the PE and FK models and a configurational one for the binary alloy) and in the thermodynamical regimes ( $h=$ const for the PE model, $c=$ const for the binary alloy and $n_{d}=$ const and/or $n_{f}=$ const for the FK model).

The single-particle Green's function for the effective atomic model (3.4) is a coherent sum of the Green's functions for subspaces $S_{i}^{z}= \pm \frac{1}{2}$ and is equal to (see Refs. $[6,13,4])$

$$
\begin{aligned}
G_{\sigma}^{(a)}\left(\omega_{\nu}\right)= & \frac{\left\langle P^{+}\right\rangle}{i \omega_{\nu}+\mu-\zeta_{\sigma}\left(\omega_{\nu}\right)-\frac{g}{2}} \\
& +\frac{\left\langle P^{-}\right\rangle}{i \omega_{\nu}+\mu-\zeta_{\sigma}\left(\omega_{\nu}\right)+\frac{g}{2}}
\end{aligned}
$$

which allows us to find solutions of Eq. (3.5) for the irreducible part $\Xi_{\sigma}\left(\omega_{\nu}\right)$ and dynamical mean field $\zeta_{\sigma}\left(\omega_{\nu}\right)$.

The Fourier transform of the two-electron Green's function $\left\langle T a_{1}^{\dagger} a_{2} a_{3}^{\dagger} a_{4}\right\rangle$ is also a coherent sum of the twoelectron Green's functions for subspaces

$$
\begin{aligned}
& G_{\sigma_{1} \sigma_{1}^{\prime}}^{\sigma \sigma^{\prime}}\left(\begin{array}{cc}
\omega_{\nu} & \omega_{\nu^{\prime}} \\
\omega_{\nu+m} & \omega_{\nu^{\prime}+m}
\end{array}\right)=\sum_{ \pm} \\
& \left\{\frac{\delta_{m 0} \delta_{\sigma \sigma_{1}} \delta_{\sigma^{\prime} \sigma_{1}^{\prime}}\left\langle P^{ \pm}\right\rangle}{\left[i \omega_{\nu}+\mu-\zeta_{\sigma}\left(\omega_{\nu}\right) \mp \frac{g}{2}\right]\left[i \omega_{\nu^{\prime}}+\mu-\zeta_{\sigma^{\prime}}\left(\omega_{\nu^{\prime}}\right) \mp \frac{g}{2}\right]}\right. \\
& \left.-\frac{\delta_{\nu \nu^{\prime}} \delta_{\sigma \sigma^{\prime}} \delta_{\sigma_{1} \sigma_{1}^{\prime}}\left\langle P^{ \pm}\right\rangle}{\left[i \omega_{\nu}+\mu-\zeta_{\sigma}\left(\omega_{\nu}\right) \mp \frac{g}{2}\right]\left[i \omega_{\nu+m}+\mu-\zeta_{\sigma_{1}}\left(\omega_{\nu+m}\right) \mp \frac{g}{2}\right]}\right\}
\end{aligned}
$$

that gives for the irreducible two-electron Green's function (3.6) an expression which is a sum of the two separable functions

$$
\begin{array}{r}
\tilde{G}_{\sigma_{1} \sigma_{1}^{\prime}}^{\sigma \sigma^{\prime}}\left(\begin{array}{cc}
\omega_{\nu} & \omega_{\nu^{\prime}} \\
\omega_{\nu+m} & \omega_{\nu^{\prime}+m}
\end{array}\right)=\delta_{m 0} \delta_{\sigma \sigma_{1}} \delta_{\sigma^{\prime} \sigma_{1}^{\prime}} \Lambda_{\sigma}\left(\omega_{\nu}\right) \Lambda_{\sigma^{\prime}}\left(\omega_{\nu^{\prime}}\right) \\
-\delta_{\nu \nu^{\prime}} \delta_{\sigma \sigma^{\prime}} \delta_{\sigma_{1} \sigma_{1}^{\prime}} \Lambda_{\sigma}\left(\omega_{\nu}\right) \Lambda_{\sigma_{1}}\left(\omega_{\nu+m}\right)
\end{array}
$$

where

$$
\Lambda_{\sigma}\left(\omega_{\nu}\right)=\frac{g \sqrt{\left\langle P^{+}\right\rangle\left\langle P^{-}\right\rangle}}{\left[i \omega_{\nu}+\mu-\zeta_{\sigma}\left(\omega_{\nu}\right)\right]^{2}-\frac{g^{2}}{4}} .
$$

Such a separable form allows us to calculate the inverse kernels of the integral equations and to find all quantities in an analytical form.
Within the weak-coupling approach, starting from the expression (4.4) with the use of Eq. (3.9), one can find solution of the Bethe-Salpeter equation for the irreducible four vertex in an analytical form:

$$
\begin{aligned}
& \Gamma_{\sigma_{1} \sigma_{1}^{\prime}}^{\sigma \sigma^{\prime}}\left(\begin{array}{cc}
\omega_{\nu} & \omega_{\nu^{\prime}} \\
\omega_{\nu+m} & \omega_{\nu^{\prime}+m}+m
\end{array}\right)=\frac{\Lambda_{\sigma}\left(\omega_{\nu}\right)}{G_{\sigma}^{2}\left(\omega_{\nu}\right)+\Lambda_{\sigma}^{2}\left(\omega_{\nu}\right)} \\
& \quad \times \frac{\delta_{m 0} \delta_{\sigma \sigma_{1}} \delta_{\sigma^{\prime} \sigma_{1}^{\prime}}}{1-\sum_{\nu^{\prime \prime} \sigma^{\prime \prime}} \frac{\Lambda_{\sigma^{\prime \prime}}^{2}\left(\omega_{\nu^{\prime \prime}}\right)}{G_{\sigma^{\prime \prime}}^{2}\left(\omega_{\nu^{\prime \prime}}\right)+\Lambda_{\sigma^{\prime \prime}}^{2}\left(\omega_{\nu^{\prime \prime}}\right)}} \cdot \frac{\Lambda_{\sigma^{\prime}}\left(\omega_{\nu^{\prime}}\right)}{G_{\sigma^{\prime}}^{2}\left(\omega_{\nu^{\prime}}\right)+\Lambda_{\sigma^{\prime}}^{2}\left(\omega_{\nu^{\prime}}\right)} \\
& -\frac{\delta_{\nu \nu^{\prime}} \delta_{\sigma \sigma^{\prime}} \delta_{\sigma_{1} \sigma_{1}^{\prime}}}{G_{\sigma}\left(\omega_{\nu}\right) G_{\sigma_{1}}\left(\omega_{\nu+m}\right)} \times \\
& \quad \frac{\Lambda_{\sigma}\left(\omega_{\nu}\right) \Lambda_{\sigma_{1}}\left(\omega_{\nu+m}\right)}{G_{\sigma}\left(\omega_{\nu}\right) G_{\sigma_{1}}\left(\omega_{\nu+m}\right)+\Lambda_{\sigma}\left(\omega_{\nu}\right) \Lambda_{\sigma_{1}}\left(\omega_{\nu+m}\right)}
\end{aligned}
$$

which must be substituted into Eq. (2.8) for the full four vertex for lattice and allows to calculate electron susceptibilities, e. g., for charge susceptibility one can get

$$
\begin{gathered}
\chi_{\boldsymbol{q}}^{n n}\left(\omega_{m}\right)=\delta_{m 0} \frac{\Delta_{n}^{2}}{T-\Theta(T, \boldsymbol{q})}+K_{\boldsymbol{q}}^{n n}\left(\omega_{m}\right), \\
K_{\boldsymbol{q}}^{n n}\left(\omega_{m}\right)=\frac{1}{\beta} \sum_{\nu \sigma} \frac{1}{\chi_{\bar{\sigma} \boldsymbol{q}}^{-1}\left(\omega_{\nu}, \omega_{m}\right)-\Gamma_{\sigma}\left(\omega_{\nu}, \omega_{m}\right)},
\end{gathered}
$$

where

$$
\begin{aligned}
\Gamma_{\sigma}\left(\omega_{\nu}, \omega_{m}\right) & = \\
& \frac{\Lambda_{\sigma}\left(\omega_{\nu}\right) \Lambda_{\sigma}\left(\omega_{\nu+m}\right)}{\tilde{\chi}_{\sigma}\left(\omega_{\nu} \omega_{m}\right)\left[\Lambda_{\sigma}\left(\omega_{\nu}\right) \Lambda_{\sigma}\left(\omega_{\nu+m}\right)-\tilde{\chi}_{\sigma}\left(\omega_{\nu} \omega_{m}\right)\right]}
\end{aligned}
$$

originates from the last term in (4.6) and

$$
\begin{gathered}
\Delta_{n}= \\
\frac{1}{\beta} \sum_{\nu \sigma} \frac{\Lambda_{\sigma}\left(\omega_{\nu}\right) \tilde{\chi}_{\sigma}\left(\omega_{\nu} 0\right) \chi_{\sigma \boldsymbol{q}}\left(\omega_{\nu} 0\right)}{\tilde{\chi}_{\sigma}^{2}\left(\omega_{\nu} 0\right)+\Lambda_{\sigma}^{2}\left(\omega_{\nu}\right)\left[\chi_{\sigma \boldsymbol{q}}\left(\omega_{\nu} 0\right)-\tilde{\chi}_{\sigma}\left(\omega_{\nu} 0\right)\right]} \\
\Theta(T, \boldsymbol{q})= \\
\frac{1}{\beta} \sum_{\nu \sigma} \frac{\Lambda_{\sigma}^{2}\left(\omega_{\nu}\right)\left[\chi_{\sigma \boldsymbol{q}}\left(\omega_{\nu} 0\right)-\tilde{\chi}_{\sigma}\left(\omega_{\nu} 0\right)\right]}{\tilde{\chi}_{\sigma}^{2}\left(\omega_{\nu} 0\right)+\Lambda_{\sigma}^{2}\left(\omega_{\nu}\right)\left[\chi_{\sigma \boldsymbol{q}}\left(\omega_{\nu} 0\right)-\tilde{\chi}_{\sigma}\left(\omega_{\nu} 0\right)\right]} \\
\chi_{\sigma \boldsymbol{q}}\left(\omega_{\nu} \omega_{m}\right)=-\frac{1}{N} \sum_{\boldsymbol{k}} G_{\sigma \boldsymbol{k}}\left(\omega_{\nu}\right) G_{\sigma \boldsymbol{k}+\boldsymbol{q}}\left(\omega_{\nu+m}\right), \\
\tilde{\chi}_{\sigma}\left(\omega_{\nu} \omega_{m}\right)=-G_{\sigma}^{(a)}\left(\omega_{\nu}\right) G_{\sigma}^{(a)}\left(\omega_{\nu+m}\right) .
\end{gathered}
$$

But such a diagrammatic weak-coupling approach does not allow one to calculate pseudospin and mixed susceptibilities.

On the other hand, the strong-coupling approach allows us to calculate all susceptibilities. To do this, we calculate the irreducible many-particle Green's functions

$$
\begin{aligned}
& \left\langle T n_{\sigma}(\tau) a_{\sigma^{\prime}}^{\dagger}\left(\tau^{\prime}\right) a_{\sigma^{\prime}}\left(\tau^{\prime \prime}\right)\right\rangle_{\mathrm{irr}} \rightarrow \delta_{m 0} \sum_{\nu^{\prime}} \Lambda_{\sigma}\left(\omega_{\nu^{\prime}}\right) \Lambda_{\sigma^{\prime}}\left(\omega_{\nu}\right) \\
& -\delta_{\sigma \sigma^{\prime}}\left[\Lambda_{\sigma}\left(\omega_{\nu}\right) \Lambda_{\sigma}\left(\omega_{\nu+m}\right)+G_{\sigma}^{(a)}\left(\omega_{\nu}\right) G_{\sigma}^{(a)}\left(\omega_{\nu+m}\right)\right],
\end{aligned}
$$




$$
\begin{gathered}
\left\langle T S^{z}(\tau) a_{\sigma}^{\dagger}\left(\tau^{\prime}\right) a_{\sigma}\left(\tau^{\prime \prime}\right)\right\rangle_{\mathrm{irr}} \rightarrow \\
\delta_{m 0} \sqrt{\left\langle P^{+}\right\rangle\left\langle P^{-}\right\rangle} \Lambda_{\sigma}\left(\omega_{\nu}\right), \\
\left\langle T S^{z}(\tau) S^{z}\left(\tau^{\prime}\right)\right\rangle_{\mathrm{irr}} \rightarrow \delta_{m 0}\left\langle P^{+}\right\rangle\left\langle P^{-}\right\rangle \\
\left\langle T n_{\sigma}(\tau) n_{\sigma^{\prime}}\left(\tau^{\prime}\right)\right\rangle_{\mathrm{irr}} \rightarrow \delta_{m 0} \sum_{\nu} \Lambda_{\sigma}\left(\omega_{\nu}\right) \sum_{\nu^{\prime}} \Lambda_{\sigma^{\prime}}\left(\omega_{\nu^{\prime}}\right) \\
-\delta_{\sigma \sigma^{\prime}} \sum_{\nu}\left[\Lambda_{\sigma}\left(\omega_{\nu}\right) \Lambda_{\sigma}\left(\omega_{\nu+m}\right)+G_{\sigma}^{(a)}\left(\omega_{\nu}\right) G_{\sigma}^{(a)}\left(\omega_{\nu+m}\right)\right] \\
\left\langle T S^{z}(\tau) n_{\sigma}\left(\tau^{\prime}\right)\right\rangle_{\mathrm{irr}} \rightarrow \\
\delta_{m 0} \sqrt{\left\langle P^{+}\right\rangle\left\langle P^{-}\right\rangle} \sum_{\nu} \Lambda_{\sigma}\left(\omega_{\nu}\right)
\end{gathered}
$$

After substituting (4.14)-(4.18) into Eq. (3.16) we get for the charge susceptibility the same expression (4.7), whereas for the pseudospin and mixed susceptibilities

$$
\begin{gathered}
\chi_{\boldsymbol{q}}^{S^{z} S^{z}}\left(\omega_{m}\right)=\delta_{m 0} \frac{\Delta_{S^{z}}^{2}}{T-\Theta(T, \boldsymbol{q})} \\
=\delta_{m 0} \frac{\left\langle P^{+}\right\rangle\left\langle P^{-}\right\rangle}{T-\Theta(T, \boldsymbol{q})} \\
\chi_{\boldsymbol{q}}^{n S^{z}}\left(\omega_{m}\right)=\chi_{\boldsymbol{q}}^{S^{z} n}\left(\omega_{m}\right)=\delta_{m 0} \frac{\Delta_{S^{z}} \Delta_{n}}{T-\Theta(T, \boldsymbol{q})}
\end{gathered}
$$

where

$$
\Delta_{S z}=\sqrt{\left\langle P^{+}\right\rangle\left\langle P^{-}\right\rangle} .
$$

Expression (4.11) for $\Theta(T, \boldsymbol{q})$ coincides with the one obtained by Freericks [7] from the equations for the static susceptibilities $\left(\omega_{m}=0\right)$ derived by Brandt and Mielsch [6]. The irreducible four vertex (4.9) was also derived by Freericks and Miller [14] within the BaymKadanoff formalism and used to find the exact solution for the nonresonant Raman scattering for the FK model $[15,16]$.

\section{DISCUSSION}

For the binary alloy (Falikov-Kimball) model (4.1) expressions (4.7), (4.19) and (4.20) define the so-called isothermal susceptibilities [17]. Furthermore, the pseudospin (4.19) and mixed (4.20) susceptibilities are only static (with factor $\delta_{m 0}$ ) because the pseudospin operator $S_{i}^{z}$ commutes with the Hamiltonian and is an integral of motion. It should be noted, that the binary alloy model (4.1) can be reduced to the Ising type model with an effective multisite retarded pseudospin interactions by taking trace of the statistical operator with the Hamiltonian (4.1) over electron (fermion) variables [4]. These explains the Ising type expression obtained for the pseudospin susceptibility (4.19), but now the expression for the critical temperature $\Theta(T, \boldsymbol{q})$ is more complicated.

The expression for the charge susceptibility (4.7) contains two terms. The first one is static and can be written as

$$
\chi^{n S^{z}}\left[\chi^{S^{z} S^{z}}\right]^{-1} \chi^{S^{z} n}
$$

This describes the contribution from the pseudospin subsystem to the charge susceptibility. It gives the main contribution to the static susceptibilities. The second term $K_{\boldsymbol{q}}^{n n}\left(\omega_{m}\right)$ in (4.7) gives the pure electron response and describes the so-called isolated (Kubo) susceptibility [17]. In general, terms with the factor $\delta_{m 0}$ give the difference between the isothermal and isolated susceptibilities (see Appendix in Ref. [18]).

\section{ACKNOWLEDGEMENT}

This work was partially supported by the Fundamental Researches Fund of the Ministry of Ukraine for Science and Education (Project No 02.07/266).
[1] A. Georges, G. Kotliar, W. Krauth, M. J. Rosenberg, Rev. Mod. Phys. 68, 13 (1996).

[2] W. Metzner, D. Vollhardt, Phys. Rev. Lett. 62, 324 (1989).

[3] W. Metzner, Phys. Rev. B 43, 8549 (1991).

[4] A. M. Shvaika, Phys. Rev. B 62, 2358 (2000).

[5] V. Zlatić, B. Horvatić, Sol. State Commun. 75, 263 (1990).

[6] U. Brandt, C. Mielsch, Z. Phys. B 75, 365 (1989); 79, 295 (1990); 82, 37 (1991).

[7] J. K. Freericks, Phys. Rev. B 47, 9263 (1993).

[8] A. M. Shvaika, Physica C 341-348, 177 (2000).

[9] A. I. Larkin, Zh. Eksp. Teor. Fiz. 37, 264 (1959).

[10] V. G. Vaks, A. I. Larkin, S. A. Pikin, Zh. Eksp. Teor. Fiz. 53, 281 (1967) [Sov. Phys. JETP 26, 188 (1968)]; 53, 1089 (1967) [Sov. Phys. JETP 26, 647 (1968)].
[11] I. V. Stasyuk, A. M. Shvaika, K. V. Tabunshchyk, Acta Phys. Pol. A 97, 411 (2000).

[12] L. M. Falicov, J. C. Kimball, Phys. Rev. Lett. 22, 997 (1969).

[13] I. V. Stasyuk, A. M. Shvaika, J. Phys. Stud. 3, 177 (1999).

[14] J. K. Freericks, P. Miller, Phys. Rev. B 62, 10022 (2000).

[15] J. K. Freericks, T. P. Devereaux, Cond. Matt. Phys. (Lviv) 4, 149 (2001).

[16] J. K. Freericks, T. P. Devereaux, Phys. Rev. B 64, 125110 (2001).

[17] R. M. Wilcox, Phys. Rev. 174, 624 (1968).

[18] V. L. Aksenov, N. M. Plakida, S. Stamenkovich, Rassieianiie Neitronov Segnetoeliektrikami (Neutron Scattering by Ferroelectrics) (Energoatomizdat, Moscow, 1984) [Translated into English (World Scientific, 1989)]. 


\title{
ДИНАМІЧНІ СПРИЙНЯТЛИВОСТІ В ПІДХОДІ СИЛЬНОГО ЗВ'ЯЗКУ: ЗАГАЛЬНА СХЕМА ТА МОДЕЛЬ ФАЛІКОВА-КІМБАЛА
}

\author{
А. М. ІІІвайка \\ Інститут фізики конденсованих систем Національной академій наук Украйни \\ вул. Свениіиького, 1, Львів, 79011, Украйна
}

\begin{abstract}
У межах теорії динамічного середнього поля розвинена загальна схема до розрахунку динамічних сприйнятливостей сильнокорельованих електронних систем. Підхід грунтується на розвиненнях за електронним переносом щодо атомної межі (у межах діяграмної техніки для вузлових операторів: проекційних чи Габбарда) для безмежної розмірности простору. Як приклад розглянуто моделі Фалікова-Кімбала та спрощену псевдоспін-електронну, для яких отримано аналітичні вирази для динамічних сприйнятливостей.
\end{abstract}

